\title{
On minimal Lagrangian surfaces in the product of Riemannian two manifolds
}

\author{
Nikos Georgiou*
}

27 May 2013

\begin{abstract}
Let $\left(\Sigma_{1}, g_{1}\right)$ and $\left(\Sigma_{2}, g_{2}\right)$ be connected, complete and orientable Riemannian two manifolds. Consider the two canonical Kähler structures $\left(G \epsilon, J, \Omega^{\epsilon}\right)$ on the product 4-manifold $\Sigma_{1} \times \Sigma_{2}$ given by $G^{\epsilon}=g_{1} \oplus \epsilon g_{2}, \epsilon= \pm 1$ and $J$ is the canonical product complex structure. Thus for $\epsilon=1$ the Kähler metric $G^{+}$is Riemannian while for $\epsilon=-1, G^{-}$is of neutral signature.

We show that the metric $G^{\epsilon}$ is locally conformally flat iff the Gauss curvatures $\kappa\left(g_{1}\right)$ and $\kappa\left(g_{2}\right)$ are both constants satisfying $\kappa\left(g_{1}\right)=-\epsilon \kappa\left(g_{2}\right)$.

We also give conditions on the Gauss curvatures for which every $G^{\epsilon}$-minimal Lagrangian surface is the product $\gamma_{1} \times \gamma_{2} \subset \Sigma_{1} \times \Sigma_{2}$, where $\gamma_{1}$ and $\gamma_{2}$ are geodesics of $\left(\Sigma_{1}, g_{1}\right)$ and $\left(\Sigma_{2}, g_{2}\right)$, respectively. Finally, we explore the Hamiltonian stability of projected rank one Hamiltonian $G^{\epsilon}$-minimal surfaces.
\end{abstract}

2000 MSC: 53D12, 49Q05

\section{Introduction}

A submanifold of a symplectic manifold is said to be Lagrangian if it is half the ambient dimension and the symplectic form vanishes on it. A Lagrangian submanifold of a pseudo-Riemannian manifold is said to be minimal if it is a critical point of the volume functional associated with pseudo-Riemannian metric. A minimal submanifold is characterized by the vanishing of the trace of its second fundamental form, the mean curvature. Recently, interest in minimal Lagrangian submanifolds in

\footnotetext{
*The author is supported by Fapesp (2010/08669-9)
} 
pseudo-Riemannian Kähler structures has grown amongst geometers [2] [22], while minimal Lagrangian submanifolds in Calabi-Yau manifolds are of great interest in theoretical physics because of their close relationship to mirror symmety [21].

In addition, the space $\mathbb{L}\left(\mathbb{M}^{3}\right)$ of oriented geodesics in a 3-dimensional space form $\left(\mathbb{M}^{3}, g\right)$ admits a natural kähler structure where the metric $G$ is of neutral signature, scalar flat and locally conformally flat [1],[3][13] [14].

The significance of these structures is that the identity component of the isometry group of $G$ is isomorphic with the identity component of the isometry group of $g$. Moreover, Salvai has proved that the neutral Kähler metrics on $\mathbb{L}\left(\mathbb{E}^{3}\right)$ and $\mathbb{L}\left(\mathbb{H}^{3}\right)$ are the unique metrics with this property [18],[19].

The neutral Kähler structure on $\mathbb{L}\left(\mathbb{M}^{3}\right)$ plays an important role in surface theory in $\left(\mathbb{M}^{3}, g\right)$. In particular, if $S$ is a smoothly immersed surface in $M$, the set of oriented geodesics normal to $S$ form a Lagrangian surface in $\mathbb{L}\left(\mathbb{M}^{3}\right)$. A Lagrangian surface $\Sigma$ in $\mathbb{L}\left(\mathbb{M}^{3}\right)$ is $G$-minimal if and only if $\Sigma$ is locally the set of normal oriented geodesics of an equidistant tube along a geodesic in $M$ [3] [6] [12].

Oh in [16] has introduced a natural variational problem, apart from the classical variational problem of minimizing the volume functional in a homology class, consisting of minimizing the volume with respect to Hamiltonian compactly supported variations. An important property of these variations is that they preserve the Lagrangian constraint. A Lagrangian submanifold in a Kähler or a pseudo-Kähler manifold is said to be Hamiltonian minimal submanifold if it is a critical point of the volume functional with respect to Hamiltonian compactly supported variations. A Hamiltonian minimal submanifold can be characterized by its mean curvature vector being divergence-free.

For example, in the space $\mathbb{L}\left(\mathbb{E}^{3}\right)$ of oriented lines in the Euclidean 3-space, a Hamiltonian minimal surface is the set of oriented lines normal to a surface $S \subset \mathbb{E}^{3}$ that is a critical point of the functional

$$
\mathcal{F}(S)=\int_{S} \sqrt{H^{2}-K} d A
$$

where $H, K$ denote the mean and the Gauss curvature of $S$, respectively [6].

The neutral Kähler structures on the space of oriented great circles in the three sphere $\mathbb{S}^{3}$ and the space of oriented space-like geodesics in the anti De Sitter 3space $A d \mathbb{S}^{3}$ can both be identified with the product structures, $\mathbb{L}\left(\mathbb{S}^{3}\right)=\mathbb{S}^{2} \times \mathbb{S}^{2}$ and $\mathbb{L}^{+}\left(\operatorname{Ad} \mathbb{S}^{3}\right)=\mathbb{H}^{2} \times \mathbb{H}^{2}$.

More generally, one is led to consider the Kähler structures derived by the product structure of $\Sigma_{1} \times \Sigma_{2}$, where $\left(\Sigma_{1}, g_{1}\right)$ and $\left(\Sigma_{2}, g_{2}\right)$ are complete, connected, orientable Riemannian 2-manifolds. 
Let $\omega_{1}$ and $\omega_{2}$ be the symplectic two forms of $\left(\Sigma_{1}, g_{1}\right)$ and $\left(\Sigma_{2}, g_{2}\right)$ respectively, and let $j_{1}$ and $j_{2}$ be their complex structures as Riemann surfaces.

For $\epsilon=1$ or -1 , consider the product structures of the four-dimensional manifold $\Sigma_{1} \times \Sigma_{2}$ endowed with the product metrics $G^{\epsilon}=\pi_{1}^{*} g_{1}+\epsilon \pi_{1}^{*} g_{2}$, the almost complex structure $J=j_{1} \oplus j_{2}$ and the symplectic two forms $\Omega^{\epsilon}=\pi_{1}^{*} \omega_{1}+\epsilon \pi_{2}^{*} \omega_{2}$, where $\pi_{i}$ are the projections of $\Sigma_{1} \times \Sigma_{2}$ onto $\Sigma_{i}, i=1,2$. The quadruples $\left(\Sigma_{1} \times \Sigma_{2}, G^{\epsilon}, J, \Omega^{\epsilon}\right)$ are easily seen to be 4-dimensional Kähler structures.

In this paper we study $G^{\epsilon}$-minimal Lagrangian surfaces in the Kähler 4-manifold $\left(\Sigma_{1} \times \Sigma_{2}, G^{\epsilon}, J, \Omega^{\epsilon}\right)$. In section 1 we prove:

Theorem 1. The Kähler metric $G^{+}$is Riemannian while the Kähler metric $G^{-}$ is neutral. Moreover, the Kähler metric $G^{\epsilon}$ is conformally flat if and if the Gauss curvatures $\kappa\left(g_{1}\right)$ and $\kappa\left(g_{2}\right)$ are both constants with $\kappa\left(g_{1}\right)=-\epsilon \kappa\left(g_{2}\right)$.

In section 2, we first define the projected rank (see Definition 1) of a surface in $\Sigma_{1} \times \Sigma_{2}$ and we prove that every Lagrangian surface is either of projected rank one or of projected rank two.

For the projected rank one case, we classify all Hamiltonian $G^{\epsilon}$-minimal surfaces:

Theorem 2. Every projected rank one Lagrangian surface can be locally parametrised by $\Phi: S \rightarrow \Sigma_{1} \times \Sigma_{2}:(s, t) \mapsto(\phi(s), \psi(t))$, where $\phi$ and $\psi$ are regular curves on $\Sigma$ and the induced metric $\Phi^{*} G^{\epsilon}$ is flat. $\Phi$ is Hamiltonian $G^{\epsilon}$-minimal if and only if $\phi$ and $\psi$ are Cornu spirals of parameters $\lambda_{\phi}$ and $\lambda_{\psi}$, respectively, such that

$$
\lambda_{\phi}=-\epsilon \lambda_{\psi}
$$

$\Phi$ is $G^{\epsilon}$-minimal Lagrangian if and only if both $\phi$ and $\psi$ are geodesics. Furthermore, every projected rank one $G^{\epsilon}$-minimal Lagrangian surface in $\Sigma_{1} \times \Sigma_{2}$ is totally geodesic.

In the same section, the following theorem gives the conditions for the non-existence of projected rank two $G^{\epsilon}$-minimal Lagrangian surfaces:

Theorem 3. Let $\left(\Sigma_{1}, g_{1}\right)$ and $\left(\Sigma_{2}, g_{2}\right)$ be Riemannian two manifolds and let $\left(G^{\epsilon}, J, \Omega^{\epsilon}\right)$ be the canonical Kähler product structures on $\Sigma_{1} \times \Sigma_{2}$. Let $\kappa\left(g_{1}\right), \kappa\left(g_{2}\right)$ be the Gauss curvatures of $g_{1}$ and $g_{2}$ respectively. Assume that either of the following hold:

(i) The metrics $g_{1}$ and $g_{2}$ are both generically non-flat and $\epsilon \kappa\left(g_{1}\right) \kappa\left(g_{2}\right)<0$ away from flat points.

(ii) Only one of the metrics $g_{1}$ and $g_{2}$ is flat while the other is non-flat generically. Then every $G^{\epsilon}$-minimal Lagrangian surface is of projected rank one. 
Here a generic property is one that holds almost everywhere. Note that Theorem 3 is no longer true when $\left(\Sigma_{1}, g_{1}\right)$ and $\left(\Sigma_{2}, g_{2}\right)$ are both flat, since there exist projected rank two minimal Lagrangian immersions in the complex Euclidean space $\mathbb{C}^{2}$ endowed with the pseudo-Hermitian product structure [6].

Minimality is the first order condition for a submanifold to be volume-extremizing in its homology class. Harvey and Lawson [15] have proven that minimal Lagrangian submanifolds of a Calabi-Yau manifold is calibrated, which implies by Stokes theorem, that are volume-extremizing. The second order condition for a minimal submanifold to be volume-extremizing was first derived by Simons [20].

Minimal submanifolds that are local extremizers of the volume are called stable minimal submanifolds. The stability of a minimal submanifold is determined by the monotonicity of the second variation of the volume functional. If the second variation of the volume functional of a Hamiltonian minimal submanifold is monotone for any Hamiltonian compactly supported variation, it is said to be Hamiltonian stable. In [16] and [17], the second variation formula of a Hamiltonian minimal submanifold has been derived in the case of a Kähler manifold, while for the pseudo-Kähler case it has been derived in [5].

The following Theorem in the section 3, investigates the Hamiltonian stability of projected rank one Hamiltonian $G^{\epsilon}$-minimal surfaces in $\Sigma_{1} \times \Sigma_{2}$ :

Theorem 4. Let $\Phi=(\phi, \psi)$ be of projected rank one Hamiltonian $G^{\epsilon}$-minimal immersion in $\left(\Sigma_{1} \times \Sigma_{2}, G^{\epsilon}\right)$ such that $\kappa\left(g_{1}\right) \leq-2 k_{\phi}^{2}$ and $\kappa\left(g_{2}\right) \leq-2 k_{\psi}^{2}$ along the curves $\phi$ and $\psi$ respectively. Then $\Phi$ is a local minimizer of the volume in its Hamiltonian isotopy class.

Acknowledgements. The author would like to thank H. Anciaux and B. Guilfoyle for their helpful and valuable suggestions and comments.

\section{The Product Kähler structure}

Consider the Riemannian 2-manifolds $\left(\Sigma_{k}, g_{k}\right)$ for $k=1,2$ and denote by $j_{k}$ the rotation by an angle $+\pi / 2$ in $T \Sigma_{k}$. Set $\omega_{k}(\cdot, \cdot)=g_{k}\left(j_{k} \cdot, \cdot\right)$ so that the quadruples $\left(\Sigma_{k}, g_{k}, j_{k}, \omega_{k}\right)$ are 2-dimensional Kähler manifolds.

Using the following identification,

$$
X \in T\left(\Sigma_{1} \times \Sigma_{2}\right) \simeq\left(X_{1}, X_{2}\right) \in T \Sigma_{1} \oplus T \Sigma_{2}, \quad \text { where } \quad X_{k} \in T \Sigma_{k} .
$$


we obtain the natural splitting $T\left(\Sigma_{1} \times \Sigma_{2}\right)=T \Sigma_{1} \oplus T \Sigma_{2}$. Let $(x, y) \in \Sigma_{1} \times \Sigma_{2}$ and $X=\left(X_{1}, X_{2}\right)$ and $Y=\left(Y_{1}, Y_{2}\right)$ be two tangent vectors in $T_{(x, y)}\left(\Sigma_{1} \times \Sigma_{2}\right)$. Define the metric $G_{(x, y)}^{\epsilon}$ by:

$$
G_{(x, y)}^{\epsilon}(X, Y)=g_{1}\left(X_{1}, Y_{1}\right)(x)+\epsilon g_{2}\left(X_{2}, Y_{2}\right)(y)
$$

where $\epsilon=1$ or -1 . The Levi-Civita connection $\nabla$ with respect to the metric $G^{\epsilon}$ is

$$
\nabla_{X} Y=\left(D_{X_{1}}^{1} Y_{1}, D_{X_{2}}^{2} Y_{2}\right)
$$

where $X=\left(X_{1}, X_{2}\right), Y=\left(Y_{1}, Y_{2}\right)$ are vector fields in $\Sigma_{1} \times \Sigma_{2}$ and $D^{1}, D^{2}$ denote the Levi-Civita connections with respect to $g_{1}$ and $g_{2}$, respectively.

Consider the endomorphism $J \in \operatorname{End}\left(T \Sigma_{1} \oplus T \Sigma_{2}\right)$ defined by $J=j_{1} \oplus j_{2}$, i.e., $J(X)=\left(j_{1} X_{1}, j_{2} X_{2}\right)$. Clearly, $J$ is an almost complex structure on $\Sigma_{1} \times \Sigma_{2}$.

Proposition 1. The almost complex structure $J$ is integrable.

Proof. The Nijenhuis tensor $N_{J}$ is

$$
N_{J}(X, Y)=[J X, J Y]^{\nabla}-J[J X, Y]^{\nabla}-J[J X, Y]^{\nabla}-[X, Y]^{\nabla},
$$

where $X=\left(X_{1}, X_{2}\right), Y=\left(Y_{1}, Y_{2}\right)$ are vector fields in $\Sigma_{1} \times \Sigma_{2}$ and $[\cdot, \cdot]^{\nabla}$ denotes the Lie bracket with respect to the Levi-Civita connection $\nabla$. Then

$$
[X, Y]^{\nabla}=\left(\left[X_{1}, Y_{1}\right]^{D^{1}},\left[X_{2}, Y_{2}\right]^{D^{2}}\right)
$$

where $[\cdot, \cdot]^{D^{i}}$ are the Lie brackets with respect to the Levi-Civita connections $D^{i}$. Thus,

$$
\begin{aligned}
N_{J}(X, Y) & =[J X, J Y]^{\nabla}-J[J X, Y]^{\nabla}-J[J X, Y]^{\nabla}-[X, Y]^{\nabla} \\
& =\left(N_{j_{1}}\left(X_{1}, Y_{1}\right), N_{j_{2}}\left(X_{2}, Y_{2}\right)\right),
\end{aligned}
$$

and the Proposition follows.

Let $\pi_{i}: \Sigma_{1} \times \Sigma_{2} \rightarrow \Sigma_{i}$ be the $i$-th projection, and define the following two-forms

$$
\Omega^{\epsilon}=\pi_{1}^{*} \omega_{1}+\epsilon \pi_{2}^{*} \omega_{2} .
$$

Theorem 1. The quadruples $\left(\Sigma_{1} \times \Sigma_{2}, G^{\epsilon}, J, \Omega^{\epsilon}\right)$ are 4-dimensional Kähler structures.

The Kähler metric $G^{\epsilon}$ is conformally flat if and if the Gauss curvatures $\kappa\left(g_{1}\right)$ and $\kappa\left(g_{2}\right)$ are both constants with $\kappa\left(g_{1}\right)=-\epsilon \kappa\left(g_{2}\right)$. 
Proof. We have already seen that the almost complex structure $J$ is integrable. It is obvious that $\Omega^{\epsilon}$ is closed, i.e., $d \Omega^{\epsilon}=0$ and is therefore a symplectic form on $\Sigma_{1} \times \Sigma_{2}$. Moreover, $J$ is compatible with $\Omega^{\epsilon}$ since for $X=\left(X_{1}, X_{2}\right)$ and $Y=\left(Y_{1}, Y_{2}\right)$, we have

$$
\begin{aligned}
\Omega_{(x, y)}^{\epsilon}(J X, J Y) & =\Omega_{(x, y)}^{\epsilon}\left(\left(j_{1} X_{1}, j_{1} X_{2}\right),\left(j_{2} Y_{1}, j_{2} Y_{2}\right)\right) \\
& =\omega_{1}\left(j_{1} X_{1}, j_{1} Y_{1}\right)(x)+\epsilon \omega_{2}\left(j_{2} X_{2}, j_{2} Y_{2}\right)(y) \\
& =\omega_{1}\left(X_{1}, Y_{1}\right)(x)+\epsilon \omega_{2}\left(X_{2}, Y_{2}\right)(y) \\
& =\Omega_{(x, y)}^{\epsilon}(X, Y) .
\end{aligned}
$$

We proceed with the proof by considering the cases of $G^{+}$and $G^{-}$.

The case of $G^{+}$: Assume that $\left(e_{1}, e_{2}\right)$ and $\left(v_{1}, v_{2}\right)$ are orthonormal frames on $\Sigma_{1}$ and $\Sigma_{2}$ respectively, both oriented in such a way $j_{1} e_{1}=e_{2}$ and $j_{2} v_{1}=v_{2}$. Consider the orthonormal frame $\left(E_{1}, E_{2}, E_{3}, E_{4}\right)$ of $\left(\Sigma_{1} \times \Sigma_{2}, G^{+}\right)$defined by

$$
\begin{array}{rlrl}
E_{1}=\frac{1}{\sqrt{3}}\left(e_{1}, v_{1}+v_{2}\right), & E_{2}=J E_{1} & =\frac{1}{\sqrt{3}}\left(e_{2}, v_{2}-v_{1}\right) \\
E_{3}=\frac{1}{\sqrt{3}}\left(e_{1}-e_{2},-v_{1}\right), & E_{4}=J E_{3}=\frac{1}{\sqrt{3}}\left(e_{1}+e_{2},-v_{2}\right) .
\end{array}
$$

If $\mathrm{Ric}^{+}$denotes the Ricci curvature tensor with respect to the metric $G^{+}$, we have

$$
\begin{aligned}
& \operatorname{Ric}^{+}\left(E_{1}, E_{1}\right)_{(x, y)}=\operatorname{Ric}^{+}\left(E_{2}, E_{2}\right)_{(x, y)}=\frac{\kappa\left(g_{1}\right)(x)+2 \kappa\left(g_{2}\right)(y)}{3}, \\
& \operatorname{Ric}^{+}\left(E_{3}, E_{3}\right)_{(x, y)}=\operatorname{Ric}^{+}\left(E_{4}, E_{4}\right)_{(x, y)}=\frac{2 \kappa\left(g_{1}\right)(x)+\kappa\left(g_{2}\right)(y)}{3},
\end{aligned}
$$

and therefore the scalar curvatute $R^{+}$is:

$$
R^{+}=\sum_{i=1}^{4} \operatorname{Ric}^{+}\left(E_{i}, E_{i}\right)=2\left(\kappa\left(g_{1}\right)(x)+\kappa\left(g_{2}\right)(y)\right) .
$$

If $G^{\epsilon}$ is conformally flat, it is scalar flat [11] and thus, from (1), the Gauss curvatures $\kappa\left(g_{1}\right), \kappa\left(g_{2}\right)$ are constants with $\kappa\left(g_{1}\right)=-\kappa\left(g_{2}\right)$.

Conversely suppose that

$$
\kappa\left(g_{1}\right)=-\kappa\left(g_{2}\right)=c,
$$

where $c$ is a real constant. Consider the corresponding coframe $\mathcal{B}_{+}=\left(e_{1}, e_{2}, e_{3}, e_{4}\right)$ of the orthonormal frame $\left(E_{1}, E_{2}, E_{3}, E_{4}\right)$. The Hodge star operator $*: \Lambda^{2}\left(\Sigma_{1} \times \Sigma_{2}\right) \rightarrow$ $\Lambda^{2}\left(\Sigma_{1} \times \Sigma_{2}\right)$ defined by

$$
a \wedge * b=G^{+}(a, b) \mathrm{Vol}
$$


splits the bundle of 2 -forms $\Lambda^{2}\left(\Sigma_{1} \times \Sigma_{2}\right)$ into:

$$
\Lambda^{2}\left(\Sigma_{1} \times \Sigma_{2}\right)=\Lambda_{+}^{2}\left(\Sigma_{1} \times \Sigma_{2}\right) \oplus \Lambda_{-}^{2}\left(\Sigma_{1} \times \Sigma_{2}\right),
$$

where $\Lambda_{+}^{2}\left(\Sigma_{1} \times \Sigma_{2}\right), \Lambda_{-}^{2}\left(\Sigma_{1} \times \Sigma_{2}\right)$ are the self-dual and the anti-self-dual 2-form bundles, respectively and $\mathrm{Vol}=e_{1} \wedge e_{2} \wedge e_{3} \wedge e_{4}$ is the volume element.

With respect to this splitting the Riemann curvature operator $\mathcal{R}: \Lambda^{2}\left(\Sigma_{1} \times \Sigma_{2}\right) \rightarrow$ $\Lambda^{2}\left(\Sigma_{1} \times \Sigma_{2}\right)$ defined by

$$
\mathcal{R}\left(e_{i} \wedge e_{j}\right) e_{k} \wedge e_{l}=G\left(R\left(E_{i}, E_{j}\right) E_{k}, E_{l}\right)
$$

is decomposed by:

$$
\mathcal{R}=\left(\begin{array}{cc}
W^{+}+\frac{R^{+}}{12} I & Z \\
Z^{*} & W^{-}+\frac{R^{+}}{12} I
\end{array}\right),
$$

where $W^{ \pm}: \Lambda_{ \pm}^{2}\left(\Sigma_{1} \times \Sigma_{2}\right) \rightarrow \Lambda_{ \pm}^{2}\left(\Sigma_{1} \times \Sigma_{2}\right)$ are the self-dual and the anti-self-dual part of the Weyl tensor $W$ and $Z$ is the traceless Ricci tensor. Note that $W=W^{+} \oplus W^{-}$. An orthonormal basis for $\Lambda_{ \pm}^{2}\left(\Sigma_{1} \times \Sigma_{2}\right)$ is

$$
\begin{aligned}
& e_{1}^{ \pm}=\frac{1}{\sqrt{2}}\left(e_{1} \wedge e_{2} \pm e_{3} \wedge e_{4}\right), \\
& e_{2}^{ \pm}=\frac{1}{\sqrt{2}}\left(e_{1} \wedge e_{3} \mp e_{2} \wedge e_{4}\right), \\
& e_{3}^{ \pm}=\frac{1}{\sqrt{2}}\left(e_{1} \wedge e_{4} \pm e_{2} \wedge e_{3}\right) .
\end{aligned}
$$

The fact that $G^{+}$is scalar flat, the self-dual part $W^{+}$vanishes since,

$$
W^{+}=R^{+}\left(\begin{array}{ccc}
1 / 3 & & \\
& -1 / 6 & \\
& & -1 / 6
\end{array}\right) \text {. }
$$

Substituting (2) into (1) the scalar curvature $R^{+}$vanishes and thus $W^{-}\left(e_{i}^{-}, e_{j}^{-}\right)=$ $\mathcal{R}\left(e_{i}^{-}\right) e_{j}^{-}$. A brief computation shows that $\mathcal{R}\left(e_{i}^{-}\right) e_{j}^{-}=0$ for all $i, j$. For example,

$$
\begin{aligned}
\mathcal{R}\left(e_{1}^{-}\right) e_{2}^{-} & =\frac{1}{2} \mathcal{R}\left(e_{1} \wedge e_{2}\right) e_{1} \wedge e_{2}+\frac{1}{2} \mathcal{R}\left(e_{3} \wedge e_{4}\right) e_{3} \wedge e_{4} \\
& =\frac{1}{2}\left(G^{+}\left(R\left(E_{1}, E_{2}\right) E_{1}, E_{2}\right)+G^{+}\left(R\left(E_{3}, E_{4}\right) E_{3}, E_{4}\right)\right) \\
& =0
\end{aligned}
$$


Thus, the anti-self-dual part $W^{-}$also vanishes. Therefore the Weyl tensor $W=0$, or $G^{+}$is locally conformally flat.

The case of $G^{-}$: We now prove that the neutral Kähler metric $G^{-}$is conformally flat if and only if the Gauss curvatures $\kappa\left(g_{1}\right), \kappa\left(g_{2}\right)$ are both constants with $\kappa\left(g_{1}\right)=$ $\kappa\left(g_{2}\right)$. For this metric, consider the orthonormal frame $\left(E_{1}, E_{2}, E_{3}, E_{4}\right)$ defined by:

$$
\begin{array}{ll}
E_{1}=\left(e_{1}, v_{1}+v_{2}\right), & E_{2}=J E_{1}=\left(e_{2}, v_{2}-v_{1}\right), \\
E_{3}=\left(e_{1}-e_{2}, v_{1}\right), & E_{4}=J E_{3}=\left(e_{1}+e_{2}, v_{2}\right) .
\end{array}
$$

In particular,

$$
-\left|E_{1}\right|^{2}=-\left|E_{2}\right|^{2}=\left|E_{3}\right|^{2}=\left|E_{4}\right|^{2}=1, \quad G\left(E_{i}, E_{j}\right)=0, \quad \forall i \neq i .
$$

A brief computation gives

$$
\begin{aligned}
& \operatorname{Ric}^{-}\left(E_{1}, E_{1}\right)=\operatorname{Ric}^{-}\left(E_{2}, E_{2}\right)=\kappa\left(g_{1}\right)(x)+2 \kappa\left(g_{2}\right)(y), \\
& \operatorname{Ric}^{-}\left(E_{3}, E_{3}\right)=\operatorname{Ric}^{-}\left(E_{4}, E_{4}\right)=2 \kappa\left(g_{1}\right)(x)+\kappa\left(g_{2}\right)(y),
\end{aligned}
$$

where $R^{-}$is the Ricci tensor of the metric $G^{-}$. Then, if $R^{-}$denotes the scalar curvature of $G^{-}$, we have

$$
\begin{aligned}
R^{-} & =\sum_{k=1}^{2}\left(-\operatorname{Ric}^{-}\left(E_{k}, E_{k}\right)+\operatorname{Ric}^{-}\left(E_{2+k}, E_{2+k}\right)\right) \\
& =2\left(\kappa\left(g_{1}\right)(x)-\kappa\left(g_{2}\right)(y)\right) .
\end{aligned}
$$

If the neutral Kähler metric $G^{-}$is conformally flat, it is also scalar flat [7] and hence, from (3), the Gauss curvatures $\kappa\left(g_{1}\right)$ and $\kappa\left(g_{2}\right)$ are constants with $\kappa\left(g_{1}\right)=\kappa\left(g_{2}\right)$. Following the same argument as before, assume the converse. That is, $\kappa\left(g_{1}\right)=$ $\kappa\left(g_{2}\right)=c$, where $c$ is a real constant. Consider the corresponding coframe $\mathcal{B}_{2}=$ $\left(e_{1}, e_{2}, e_{3}, e_{4}\right)$ and the Hodge star operator $*: \Lambda^{2}\left(\Sigma_{1} \times \Sigma_{2}\right) \rightarrow \Lambda^{2}\left(\Sigma_{1} \times \Sigma_{2}\right)$.

The Hodge star operator splits the Riemann curvature operator $\mathcal{R}: \Lambda^{2}\left(\Sigma_{1} \times \Sigma_{2}\right) \rightarrow$ $\Lambda^{2}\left(\Sigma_{1} \times \Sigma_{2}\right)$ in the same way as in the Riemannian case. The Weyl $(0,4)$-tensor $W$ is given by:

$$
W_{i j k l}=R_{i j k l}^{G}-\frac{1}{2}\left(-G_{j k} R i c_{i l}^{G}+G_{j l} R i c_{i k}^{G}-G_{i l} R i c_{j k}^{G}+G_{i k} R i c_{j l}^{G}\right),
$$


where $R_{i j k l}^{G}=G\left(R^{G}\left(E_{i}, E_{j}\right) E_{k}, E_{l}\right)$. An orthonormal basis for $\Lambda_{ \pm}^{2}\left(\Sigma_{1} \times \Sigma_{2}\right)$, in the neutral case, is

$$
\begin{aligned}
& e_{1}^{ \pm}=\frac{1}{\sqrt{2}}\left(e_{1} \wedge e_{2} \pm e_{3} \wedge e_{4}\right), \\
& e_{2}^{ \pm}=\frac{1}{\sqrt{2}}\left(e_{1} \wedge e_{3} \pm e_{2} \wedge e_{4}\right), \\
& e_{3}^{ \pm}=\frac{1}{\sqrt{2}}\left(e_{1} \wedge e_{4} \mp e_{2} \wedge e_{3}\right) .
\end{aligned}
$$

The fact that $G^{-}$is scalar flat, following [7], the anti-self-dual part $W^{-}$vanishes since,

$$
W^{-}=R^{-}\left(\begin{array}{ccc}
1 / 3 & & \\
& 1 / 6 & \\
& & 1 / 6
\end{array}\right)
$$

The self-dual part is

$$
W^{+}=\left(\begin{array}{ccc}
W_{1212}+W_{3434}+2 W_{1234} & 2\left(W_{1213}+W_{1334}\right) & 2\left(W_{1214}+W_{1434}\right) \\
& 2\left(W_{1313}+W_{1324}\right) & 2\left(W_{1314}-W_{1323}\right) \\
& & 2\left(W_{1414}-W_{1423}\right)
\end{array}\right)
$$

and a brief computation shows that $W^{+}$vanishes. Therefore, the Weyl tensor $W$ vanishes, or $G$ is locally conformally flat.

Corollary 1. Let $(\Sigma, g)$ be a Riemannian two manifold. The neutral Kähler metric $G^{-}$of the four dimensional Kähler manifold $\Sigma \times \Sigma$ is conformally flat if and only if the metric $g$ is of constant Gaussian curvature.

\section{Surface theory in the 4-manifold $\Sigma_{1} \times \Sigma_{2}$}

Let $\Phi: S \rightarrow \Sigma_{1} \times \Sigma_{2}$ be a smooth immersion of a surface $S$ in $\Sigma_{1} \times \Sigma_{2}$, where $\left(\Sigma_{1}, g_{1}\right)$ and $\left(\Sigma_{2}, g_{2}\right)$ are both Riemannian two manifolds and let $\pi_{i}$ be the projections of $\Sigma_{1} \times \Sigma_{2}$ onto $\Sigma_{i}, i=1,2$. We denote by $\phi$ and $\psi$ the mappings $\pi_{1} \circ \Phi$ and $\pi_{2} \circ \Phi$, respectively and we write $\Phi=(\phi, \psi)$. The rank of a mapping at a point is the rank of its derivative at that point. 
Definition 1. The immersion $\Phi=(\phi, \psi): S \rightarrow \Sigma_{1} \times \Sigma_{2}$ is said to be of projected rank zero at a point $p \in S$ if either $\operatorname{rank}(\phi(p))=0$ or $\operatorname{rank}(\psi(p))=0$. $\Phi$ is of projected rank one at $p$ if either $\operatorname{rank}(\phi(p))=1$ or $\operatorname{rank}(\psi(p))=1$. Finally, $\Phi$ is of projected rank two at $p$ if $\operatorname{rank}(\phi(p))=\operatorname{rank}(\psi(p))=2$.

Note that, since it is an immersion, $\Phi$ must be of projected rank zero, one or two.

\subsection{Projected rank zero case}

Let $\Phi=(\phi, \psi)$ be of projected rank zero immersion in $\Sigma_{1} \times \Sigma_{2}$. Assuming, without loss of generality, that $\operatorname{rank}(\phi)=0$, the map $\phi$ is locally a constant function and the map $\psi$ is a local diffeomorphism. We now give the following Proposition:

Proposition 2. There are no Lagrangian immersions in $\Sigma_{1} \times \Sigma_{2}$ of projected rank zero.

Proof. If $\Phi=(\phi, \psi): S \rightarrow \Sigma_{1} \times \Sigma_{2}$ were an immersed surface with $\operatorname{rank}(\phi)=0$, then $\psi: S \rightarrow \Sigma_{2}$ is a local diffeomorphism and thus for any vector fields $X, Y$ on $S$

$$
\begin{aligned}
\Phi^{*} \Omega^{\epsilon}(X, Y) & =\Omega^{\epsilon}(d \Phi(X), d \Phi(Y)) \\
& =\Omega^{\epsilon}((0, d \psi(X)),(0, d \psi(Y))) \\
& =\epsilon \omega(d \psi(X), d \psi(Y)) \\
& \neq 0,
\end{aligned}
$$

where the last line follows from the non-degeneracy of $\omega$ and the fact that $d \psi$ is a bundle isomorphism.

\subsection{Projected rank one Lagrangian surfaces}

We begin by giving a definition of the Cornu spirals in a Riemannian two manifold.

Definition 2. Let $(\Sigma, g)$ be a Riemannian two manifold. A regular curve $\gamma$ of $\Sigma$ is called a Cornu spiral of parameter $\lambda$ if its curvature $\kappa_{\gamma}$ is a linear function of its arclength parameter such that $\kappa_{\gamma}(s)=\lambda s+\mu$, where $s$ is the arclength and $\lambda, \mu$ are real constants. 
A Cornu spiral $\gamma$ in $\mathbb{R}^{2}$ of parameter $\lambda$ can be parametrised, up to congruences, by

$$
\gamma(s)=\left(\int_{0}^{s} \cos \left(\lambda t^{2} / 2\right) d t, \int_{0}^{s} \sin \left(\lambda t^{2} / 2\right) d t\right)
$$

and they are bounded but have infinite length [4].

Let $\Phi=(\phi, \psi): S \rightarrow \Sigma_{1} \times \Sigma_{2}$ be of projected rank one immersion in $\Sigma_{1} \times \Sigma_{2}$. Then either $\phi$ or $\psi$ is of rank one. The following theorem gives all rank one Hamiltonian $G^{\epsilon}$-minimal surfaces:

Theorem 2. Every projected rank one Lagrangian surface can be locally parametrised by $\Phi: S \rightarrow \Sigma_{1} \times \Sigma_{2}:(s, t) \mapsto(\phi(s), \psi(t))$, where $\phi$ and $\psi$ are regular curves on $\Sigma$ and the induced metric $\Phi^{*} G^{\epsilon}$ is flat. In addition, $\Phi$ is Hamiltonian $G^{\epsilon}$-minimal if and only if $\phi$ and $\psi$ are Cornu spirals of parameters $\lambda_{\phi}$ and $\lambda_{\psi}$ respectively such that

$$
\lambda_{\phi}=-\epsilon \lambda_{\psi}
$$

Moreover, $\Phi$ is $G^{\epsilon}$-minimal Lagrangian if and only if both $\phi$ and $\psi$ are geodesics, and every projected rank one $G^{\epsilon}$-minimal Lagrangian surface in $\Sigma_{1} \times \Sigma_{2}$ is totally geodesic.

Proof. Let $\Phi=(\phi, \psi): S \rightarrow \Sigma_{1} \times \Sigma_{2}$ be of projected rank one Lagrangian immersion. Assume, without loss of generality, that $\phi$ is of rank one. We now prove that $\psi$ is of rank one.

Since $\Phi$ is an immersion of a surface, the map $\psi$ cannot be of rank zero. Suppose that $\psi$ is of rank two, i.e. a local diffeomorphism. Thus, $\Phi$ is locally parametrised by $\Phi: U \subset S \rightarrow \Sigma_{1} \times \Sigma_{2}:(s, t) \mapsto(\phi(s), \psi(s, t))$. Hence,

$$
\Phi_{s}=\left(\phi^{\prime}(s), \psi_{s}\right) \quad \Phi_{t}=\left(0, \psi_{t}\right) .
$$

Since $\Phi$ is a Lagrangian immersion we have that $\omega_{2}\left(\psi_{s}, \psi_{t}\right)=0$.

The fact that $\psi$ is a local diffeomorphism implies that for any non-zera vector field $X$ in $\Sigma_{2}$ can be written as $X=a \psi_{s}+b \psi_{t}$ and therefore we have that $\omega_{2}\left(\psi_{s}, X\right)=0$. The nondegeneracy of $\omega_{2}$ implies that $\psi$ is cannot be a local diffeomorphism since $\psi_{s}=0$. Thus $\psi$ is also a rank one immersion.

We now have that $S$ is locally parametrised by $\Phi: U \subset S \rightarrow \Sigma_{1} \times \Sigma_{2}:(s, t) \mapsto$ $(\phi(s), \psi(t))$, where $\phi$ and $\psi$ are regular curves in $\Sigma_{1}$ and $\Sigma_{2}$, respectively. If $s, t$ are the corresponding arc-length parameters of $\phi$ and $\psi$, the Frénet equtions give

$$
D_{\phi^{\prime}}^{1} \phi^{\prime}=k_{\phi} j \phi^{\prime} \quad D_{\psi^{\prime}}^{2} \psi^{\prime}=k_{\psi} j \psi^{\prime},
$$


where $k_{\phi}$ and $k_{\psi}$ denote the curvatures of $\phi$ and $\psi$, respectively. Moreover, $\Phi_{s}=$ $\left(\phi^{\prime}, 0\right)$ and $\Phi_{t}=\left(0, \psi^{\prime}\right)$ and thus,

$\nabla_{\Phi_{s}} \Phi_{s}=\left(D_{\phi^{\prime}}^{1} \phi^{\prime}, 0\right)=\left(k_{\phi} j \phi^{\prime}, 0\right), \quad \nabla_{\Phi_{t}} \Phi_{t}=\left(0, D_{\psi^{\prime}}^{2} \psi^{\prime}\right)=\left(0, k_{\psi} j \psi^{\prime}\right), \quad \nabla_{\Phi_{t}} \Phi_{s}=(0,0)$.

The first fundamental form $G_{i j}^{\epsilon}=G^{\epsilon}\left(\partial_{i} \Phi, \partial_{j} \Phi\right)$ is given by

$$
G_{s s}=\epsilon G_{t t}=1, \quad G_{s t}=0
$$

which proves that the immersion $\Phi$ is flat.

The second fundamental form $h^{\epsilon}$ of $\Phi$, is completely determined by the following tri-symmetric tensor

$$
h^{\epsilon}(X, Y, Z):=G^{\epsilon}\left(h^{\epsilon}(X, Y), J Z\right)=\Omega^{\epsilon}\left(X, \nabla_{Y} Z\right)
$$

We then have,

$$
h_{s s t}^{\epsilon}=\Omega^{\epsilon}\left(\Phi_{s}, \nabla_{\Phi_{s}} \Phi_{t}\right)=0, \quad h_{s t t}^{\epsilon}=\Omega^{\epsilon}\left(\Phi_{s}, \nabla_{\Phi_{t}} \Phi_{t}\right)=0 .
$$

Moreover,

$$
h_{s s s}^{\epsilon}=\Omega^{\epsilon}\left(\Phi_{s}, \nabla_{\Phi_{s}} \Phi_{s}\right)=\Omega^{\epsilon}\left(\left(\phi^{\prime}, 0\right),\left(k_{\phi} j \phi^{\prime}, 0\right)\right)=G^{\epsilon}\left(\left(j \phi^{\prime}, 0\right),\left(k_{\phi} j \phi^{\prime}, 0\right)\right)=k_{\phi},
$$

and similarly, $h_{t t t}^{\epsilon}=\epsilon k_{\psi}$. Denote the mean curvature of $\Phi$ with respect to the metric $G^{\epsilon}$ by $\vec{H}^{\epsilon}$. Then

$$
G^{\epsilon}\left(2 \vec{H}^{\epsilon}, J \Phi_{s}\right)=\frac{h_{s s s}^{\epsilon} G_{t t}^{\epsilon}+h_{s t t}^{\epsilon} G_{s s}^{\epsilon}-2 h_{s s t}^{\epsilon} G_{s t}^{\epsilon}}{G_{s s}^{\epsilon} G_{t t}^{\epsilon}-\left(G_{s t}^{\epsilon}\right)^{2}}=k_{\phi},
$$

and

$$
G^{\epsilon}\left(2 \vec{H}^{\epsilon}, J \Phi_{t}\right)=\frac{h_{s s t}^{\epsilon} G_{t t}^{\epsilon}+h_{t t t}^{\epsilon} G_{s s}^{\epsilon}-2 h_{s t t}^{\epsilon} G_{s t}^{\epsilon}}{G_{s s}^{\epsilon} G_{t t}^{\epsilon}-\left(G_{s t}^{\epsilon}\right)^{2}}=k_{\psi}
$$

Hence

$$
2 \vec{H}^{\epsilon}=k_{\phi} J \Phi_{s}+\epsilon k_{\psi} J \Phi_{t} .
$$

It is not hard to see that the Lagrangian immersion $\Phi$ is $G^{\epsilon}$-minimal iff the curves $\phi$ and $\psi$ are geodesics. Moreover, if $\Phi$ is $G^{\epsilon}$-minimal Lagrangian it is totally geodesic since the second fundamental form vanishes identically.

Note also that,

$$
\operatorname{div}^{\epsilon}\left(\Phi_{s}\right)=-G^{\epsilon}\left(\nabla_{\Phi_{s}} \Phi_{s}, \Phi_{s}\right)=-G^{\epsilon}\left(\left(k_{\phi} j \phi^{\prime}, 0\right),\left(\phi^{\prime}, 0\right)\right)=-g\left(k_{\phi} j \phi^{\prime}, \phi^{\prime}\right)=0
$$


In a similar way, we derive that $\operatorname{div}^{\epsilon}\left(\Phi_{t}\right)=0$.

Thus,

$$
\begin{aligned}
-\operatorname{div}^{\epsilon}\left(2 J \vec{H}^{\epsilon}\right) & =G^{\epsilon}\left(\nabla k_{\phi}, \Phi_{s}\right)+k_{\phi} \operatorname{div}^{\epsilon}\left(\Phi_{s}\right)+\epsilon G^{\epsilon}\left(\nabla k_{\psi}, \Phi_{t}\right)+\epsilon k_{\psi} \operatorname{div}^{\epsilon}\left(\Phi_{t}\right) \\
& =\frac{D}{d s} k_{\phi}(s)+\epsilon \frac{D}{d t} k_{\psi}(t),
\end{aligned}
$$

and the theorem follows.

\subsection{Projected rank two Lagrangian surfaces}

For the projected rank two case, we have the following Theorem:

Theorem 3. Let $\left(\Sigma_{1}, g_{1}\right)$ and $\left(\Sigma_{2}, g_{2}\right)$ be Riemannian two manifolds and let $\left(G^{\epsilon}, J, \Omega^{\epsilon}\right)$ be the canonical Kähler product structures on $\Sigma_{1} \times \Sigma_{2}$ constructed in section 1. Let $\kappa\left(g_{1}\right), \kappa\left(g_{2}\right)$ be the Gauss curvatures of $g_{1}$ and $g_{2}$ respectively. Assume that one of the following holds:

(i) The metrics $g_{1}$ and $g_{2}$ are both generically non-flat and $\epsilon \kappa\left(g_{1}\right) \kappa\left(g_{2}\right)<0$ away from flat points.

(ii) Only one of the metrics $g_{1}$ and $g_{2}$ is flat while the other is non-flat generically. Then every $G^{\epsilon}$-minimal Lagrangian surface is of projected rank one.

Proof. Assume that the $G^{\epsilon}$-minimal Lagrangian immersion $\Phi=(\phi, \psi): S \rightarrow \Sigma_{1} \times \Sigma_{2}$ is of projected rank two. Then by definition the mappings $\phi: S \rightarrow \Sigma_{1}$ and $\psi: S \rightarrow$ $\Sigma_{2}$ are both local diffeomorphisms. The Lagrangian assumption $\Phi^{*} \Omega^{\epsilon}=0$ yields

$$
\phi^{*} \omega_{1}=-\epsilon \psi^{*} \omega_{2}
$$

Take an orthonormal frame $\left(e_{1}, e_{2}\right)$ of $\Phi^{*} G^{\epsilon}$ such that,

$$
G^{\epsilon}\left(d \Phi\left(e_{1}\right), d \Phi\left(e_{1}\right)\right)=\epsilon G^{\epsilon}\left(d \Phi\left(e_{2}\right), d \Phi\left(e_{2}\right)\right)=1, \quad G^{\epsilon}\left(d \Phi\left(e_{1}\right), d \Phi\left(e_{2}\right)\right)=0 .
$$

The Lagrangian condition implies that the frame $\left(d \Phi\left(e_{1}\right), d \Phi\left(e_{2}\right), J d \Phi\left(e_{1}\right), J d \Phi\left(e_{2}\right)\right)$ is orthonormal. Let $\left(s_{1}, s_{2}\right)$ and $\left(v_{1}, v_{2}\right)$ be oriented orthonormal frames of $\left(\Sigma_{1}, g_{1}\right)$ and $\left(\Sigma_{2}, g_{2}\right)$ respectively such that $j_{1} s_{1}=s_{2}$ and $j_{2} v_{1}=v_{2}$. Then there exist smooth functions $\lambda_{1}, \lambda_{2}, \mu_{1}, \mu_{2}$ on $\Sigma_{1}$ and $\bar{\lambda}_{1}, \bar{\lambda}_{2}, \bar{\mu}_{1}, \bar{\mu}_{2}$ on $\Sigma_{2}$ such that

$$
\begin{array}{ll}
d \phi\left(e_{1}\right)=\lambda_{1} s_{1}+\lambda_{2} s_{2} & d \phi\left(e_{2}\right)=\mu_{1} s_{1}+\mu_{2} s_{2}, \\
d \psi\left(e_{1}\right)=\bar{\lambda}_{1} v_{1}+\bar{\lambda}_{2} v_{2} & d \psi\left(e_{2}\right)=\bar{\mu}_{1} v_{1}+\bar{\mu}_{2} v_{2} .
\end{array}
$$


Thus,

$$
\phi^{*} \omega_{1}\left(e_{1}, e_{2}\right)=\lambda_{1} \mu_{2}-\lambda_{2} \mu_{1}, \quad \psi^{*} \omega_{2}\left(e_{1}, e_{2}\right)=\bar{\lambda}_{1} \bar{\mu}_{2}-\bar{\lambda}_{2} \bar{\mu}_{1}
$$

and using the Lagrangian condition (4) we have

$$
\left(\lambda_{1} \mu_{2}-\lambda_{2} \mu_{1}\right)(\phi(p))=-\epsilon\left(\bar{\lambda}_{1} \bar{\mu}_{2}-\bar{\lambda}_{2} \bar{\mu}_{1}\right)(\psi(p)), \quad \forall p \in S
$$

Moreover, the assumption that $\Phi$ is of projected rank two, implies that $\lambda_{1} \mu_{2}-\lambda_{2} \mu_{1} \neq$ 0 for every $p \in S$.

If $H^{\epsilon}$ is the mean curvature vector of the immersion $\Phi$, consider the one form $a_{H^{\epsilon}}$ defined by $a_{H^{\epsilon}}=G^{\epsilon}\left(J H^{\epsilon}, \cdot\right)$. Since $\Phi$ is Lagrangian, it is known from [9] that

$$
d a_{H^{\epsilon}}=\Phi^{*} \rho^{\epsilon}
$$

where $\rho^{\epsilon}$ is the Ricci form of $G^{\epsilon}$. The fact that $\Phi$ is a $G^{\epsilon}$-minimal Lagrangian immersion implies that $\Phi^{*} \rho^{\epsilon}$ vanishes and therefore,

$$
\begin{aligned}
0= & \rho^{\epsilon}\left(d \Phi\left(e_{1}\right), d \Phi\left(e_{2}\right)\right) \\
= & \operatorname{Ric}^{\epsilon}\left(d \Phi\left(e_{1}\right), J d \Phi\left(e_{2}\right)\right) \\
= & \epsilon G^{\epsilon}\left(R\left(d \Phi e_{1}, d \Phi e_{2}\right) J d \Phi e_{2}, d \Phi e_{2}\right)+G^{\epsilon}\left(R\left(d \Phi e_{1}, d \Phi e_{2}\right) J d \Phi e_{1}, d \Phi e_{1}\right) \\
= & \epsilon g_{1}\left(R_{1}\left(d \phi e_{1}, d \phi e_{2}\right) j_{1} d \phi e_{2}, d \phi e_{2}\right)+g_{2}\left(R_{2}\left(d \psi e_{1}, d \psi e_{2}\right) j_{2} d \psi e_{2}, d \psi e_{2}\right) \\
& \quad+g_{1}\left(R_{1}\left(d \phi e_{1}, d \phi e_{2}\right) j_{1} d \phi e_{1}, d \phi e_{1}\right)+\epsilon g_{2}\left(R_{2}\left(d \psi e_{1}, d \psi e_{2}\right) J d \psi e_{1}, d \psi e_{1}\right) . \\
= & \epsilon\left(\left(\lambda_{1}^{2}+\lambda_{2}^{2}+\epsilon\left(\mu_{1}^{2}+\mu_{2}^{2}\right)\right)\left(\mu_{1} \lambda_{2}-\mu_{2} \lambda_{1}\right) \kappa\left(g_{1}\right)\right. \\
& \quad+\left(\bar{\lambda}_{1}^{2}+\bar{\lambda}_{2}^{2}+\epsilon\left(\bar{\mu}_{1}^{2}+\bar{\mu}_{2}^{2}\right)\right)\left(\bar{\mu}_{1} \bar{\lambda}_{2}-\bar{\mu}_{2} \bar{\lambda}_{1}\right) \kappa\left(g_{2}\right) \\
& \quad \\
= & \left.\epsilon\left(\mu_{1} \lambda_{2}-\mu_{2} \lambda_{1}\right)\left[\left(\lambda_{1}^{2}+\lambda_{2}^{2}+\epsilon\left(\mu_{1}^{2}+\mu_{2}^{2}\right)\right) \kappa\left(g_{1}\right)-\left(\bar{\lambda}_{1}^{2}+\bar{\lambda}_{2}^{2}+\epsilon\left(\bar{\mu}_{1}^{2}+\bar{\mu}_{2}^{2}\right)\right) \kappa\left(g_{2}\right)\right)\right]
\end{aligned}
$$

which finally gives,

$$
\left(\lambda_{1}^{2}+\lambda_{2}^{2}+\epsilon\left(\mu_{1}^{2}+\mu_{2}^{2}\right)\right) \kappa\left(g_{1}\right)=\left(\bar{\lambda}_{1}^{2}+\bar{\lambda}_{2}^{2}+\epsilon\left(\bar{\mu}_{1}^{2}+\bar{\mu}_{2}^{2}\right)\right) \kappa\left(g_{2}\right) .
$$

The condition $G^{\epsilon}\left(d \Phi\left(e_{1}\right), d \Phi\left(e_{2}\right)\right)=0$ yields

$$
\lambda_{1} \mu_{1}+\lambda_{2} \mu_{2}=-\epsilon\left(\bar{\lambda}_{1} \bar{\mu}_{1}+\bar{\lambda}_{2} \bar{\mu}_{2}\right) .
$$

Now using (4) and (7) we have

$$
\left(\lambda_{1}^{2}+\lambda_{2}^{2}\right)\left(\mu_{1}^{2}+\mu_{2}^{2}\right)=\left(\bar{\lambda}_{1}^{2}+\bar{\lambda}_{2}^{2}\right)\left(\bar{\mu}_{1}^{2}+\bar{\mu}_{2}^{2}\right) .
$$


From $G^{\epsilon}\left(d \Phi\left(e_{1}\right), d \Phi\left(e_{1}\right)\right)=\epsilon G^{\epsilon}\left(d \Phi\left(e_{2}\right), d \Phi\left(e_{2}\right)\right)=1$ we obtain

$$
\lambda_{1}^{2}+\lambda_{2}^{2}+\epsilon\left(\bar{\lambda}_{1}^{2}+\bar{\lambda}_{2}^{2}\right)=\epsilon\left(\mu_{1}^{2}+\mu_{2}^{2}\right)+\bar{\mu}_{1}^{2}+\bar{\mu}_{2}^{2}=1 .
$$

Set $a:=\lambda_{1}^{2}+\lambda_{2}^{2}, b:=\mu_{1}^{2}+\mu_{2}^{2}, \bar{a}:=\bar{\lambda}_{1}^{2}+\bar{\lambda}_{2}^{2}, \bar{b}:=\bar{\mu}_{1}^{2}+\bar{\mu}_{2}^{2}$. The relations (7), (8) and (9) give

$$
a b=\bar{a} \bar{b} \quad a+\epsilon \bar{a}=\epsilon b+\bar{b}=1 .
$$

Thus $a=-\epsilon \bar{a}+1$ and $b=\epsilon-\epsilon \bar{b}$, and from $a b=\bar{a} \bar{b}$ we have that $\bar{a}+\epsilon \bar{b}=\epsilon$. Moreover, $\bar{a}=\epsilon-\epsilon a$ and $\bar{b}=1-\epsilon b$, and again from $a b=\bar{a} \bar{b}$ we have $a+\epsilon b=1$. Hence, relation (6) becomes

$$
\kappa\left(g_{1}\right)(\phi(p))=\epsilon \kappa\left(g_{2}\right)(\psi(p)), \quad \text { for every } p \in S,
$$

which implies that the metrics $g_{1}$ and $g_{2}$ can satisfy neither condition (i) nor condition (ii) of the statement.

The following Corollaries follow:

Corollary 2. Every $G^{+}$-minimal Lagrangian surface immersed in $\mathbb{S}^{2} \times \mathbb{H}^{2}$ is, up to isometry, the cylinder $\mathbb{S}^{1} \times \mathbb{R}$. Moreover, every $G^{\epsilon}$-minimal Lagrangian surface immersed in $\mathbb{R}^{2} \times \mathbb{H}^{2}\left(\mathbb{R}^{2} \times \mathbb{S}^{2}\right)$ is of projected rank one and therefore it is $\gamma_{1} \times \gamma_{2}$, where $\gamma_{1}$ is a straight line in $\mathbb{R}^{2}$ and $\gamma_{2}$ is a geodesic in $\mathbb{H}^{2}\left(\gamma_{2}\right.$ is a geodesic in $\left.\mathbb{S}^{2}\right)$, respectively.

Corollary 3. Let $(\Sigma, g)$ be a Riemannian two manifold such that the metric $g$ is non-flat. Then every $G^{-}$-minimal Lagrangian surface immersed in $\Sigma \times \Sigma$ is of projected rank one and is therefore the product of two geodesics of $(\Sigma, g)$.

\section{The Hamiltonian stability of minimal Lagrangian surfaces}

The Hamiltonian stability of a Hamiltonian minimal surface $S$ in a pseudo-Riemannian manifold $(\mathcal{M}, G)$ is given by the monotonicity of the second variation formula of the volume $V(S)$ under Hamiltonian deformations (see [16] and [5]). For a smooth compactly supported function $u \in C_{c}^{\infty}(S)$ the second variation $\delta^{2} V(S)(X)$ formula in the direction of the Hamiltonian vector field $X=J \nabla u$ is:

$\delta^{2} V(S)(X)=\int_{S}\left((\Delta u)^{2}-\operatorname{Ric}^{G}(\nabla u, \nabla u)-2 G(h(\nabla u, \nabla u), n H)+G^{2}(n H, J \nabla u)\right) d V$ 
where $h$ is the second fundamental form of $S, \operatorname{Ric}^{G}$ is the Ricci curvature tensor of the metric $G$, and $\Delta$ with $\nabla$ denote the Laplacian and gradient, respectively, with respect to the metric $G$ induced on $S$. For the Hamiltonian stability of projected rank one Hamiltonian $G^{\epsilon}$-minimal surfaces we give the following Theorem:

Theorem 4. Let $\Phi=(\phi, \psi)$ be of projected rank one Hamiltonian $G^{\epsilon}$-minimal immersion in $\left(\Sigma_{1} \times \Sigma_{2}, G^{\epsilon}\right)$ such that $\kappa\left(g_{1}\right) \leq-2 k_{\phi}^{2}$ and $\kappa\left(g_{2}\right) \leq-2 k_{\psi}^{2}$ along the curves $\phi$ and $\psi$ respectively. Then $\Phi$ is a local minimizer of the volume in its Hamiltonian isotopy class.

Proof. Let $\Phi=(\phi, \psi): S \rightarrow \Sigma_{1} \times \Sigma_{2}$ be of projected rank one Hamiltonian $G^{\epsilon}$ minimal immersion and let $(s, t)$ be the corresponded arclengths of $\phi$ and $\psi$, respectively.

Then $\left(\phi_{s}, j_{1} \phi_{s}\right)$ is an oriented orthonormal frame of $\left(\Sigma_{1}, g_{1}\right)$ and $\left(\psi_{t}, j_{2} \psi_{t}\right)$ is an oriented orthonormal frame of $\left(\Sigma_{2}, g_{2}\right)$.

Therefore,

$$
\begin{aligned}
\operatorname{Ric}^{\epsilon}\left(\Phi_{s}, \Phi_{s}\right) & =\epsilon^{\epsilon}\left(R\left(\Phi_{t}, \Phi_{s}\right) \Phi_{s}, \Phi_{t}\right)+G^{\epsilon}\left(R\left(J \Phi_{s}, \Phi_{s}\right) \Phi_{s}, J \Phi_{s}\right) \\
& =G^{\epsilon}\left(R\left(J \Phi_{s}, \Phi_{s}\right) \Phi_{s}, J \Phi_{s}\right) \\
& =G^{\epsilon}\left(\left(R_{1}\left(j_{1} \phi_{s}, \phi_{s}\right) \Phi_{s}, J \Phi_{t}\right)\right. \\
& =G^{\epsilon}\left(\left(R_{1}\left(j_{1} \phi_{s}, \phi_{s}\right) \phi_{s}, 0\right),\left(j_{1} \phi_{s}, 0\right)\right) \\
& =g_{1}\left(R_{1}\left(j_{1} \phi_{s}, \phi_{s}, \phi_{s}\right)\right) \\
& =\kappa\left(g_{1}\right) .
\end{aligned}
$$

Moreover, a similar computation gives

$$
\operatorname{Ric}^{\epsilon}\left(\Phi_{t}, \Phi_{t}\right)=\kappa\left(g_{2}\right) \text { and } \operatorname{Ric}^{\epsilon}\left(\Phi_{s}, \Phi_{t}\right)=0 .
$$

Then, for every $u(s, t) \in C_{c}^{\infty}(S)$ we have

$$
\operatorname{Ric}^{\epsilon}(\nabla u, \nabla u)=\kappa\left(g_{1}\right) u_{s}^{2}+\kappa\left(g_{2}\right) u_{t}^{2} .
$$

Furthermore

$$
G^{\epsilon}\left(h^{\epsilon}(\nabla u, \nabla u), 2 \vec{H}^{\epsilon}\right)=u_{s}^{2} k_{\phi}^{2}+u_{t}^{2} k_{\psi}^{2},
$$

and

$$
G^{\epsilon}\left(2 \vec{H}^{\epsilon}, J \nabla u\right)=u_{s} k_{\phi}+\epsilon u_{t} k_{\psi} .
$$


The second variation formula for the volume functional with respect of the Hamiltonian vector field $X=J \nabla u$ therefore becomes

$$
\begin{aligned}
\delta^{2} V(S)(X) & =\int_{S}\left(\Delta^{\epsilon} u\right)^{2}-\operatorname{Ric}^{\epsilon}(\nabla u, \nabla u)-2 G^{\epsilon}\left(h^{\epsilon}(\nabla u, \nabla u), 2 \vec{H}^{\epsilon}\right)+G^{\epsilon}\left(2 \vec{H}^{\epsilon}, J \nabla u\right)^{2} \\
& =\int_{S}\left(u_{s s}+\epsilon u_{t t}\right)^{2}-u_{s}^{2} \kappa\left(g_{1}\right)-u_{t}^{2} \kappa\left(g_{2}\right)-\left(u_{s} k_{\phi}-\epsilon u_{t} k_{\psi}\right)^{2} \\
& =\int_{S}\left(u_{s s}+\epsilon u_{t t}\right)^{2}+u_{s}^{2}\left(-\kappa\left(g_{1}\right)-k_{\phi}^{2}\right)+u_{t}^{2}\left(-\kappa\left(g_{2}\right)-k_{\psi}^{2}\right)+2 \epsilon u_{s} u_{t} k_{\phi} k_{\psi} .
\end{aligned}
$$

Assuming that $\kappa\left(g_{1}\right) \leq-2 k_{\phi}^{2}$ and $\kappa\left(g_{2}\right) \leq-2 k_{\psi}^{2}$ along the curves $\phi$ and $\psi$, respectively we conclude that the second variation formula is nonnegative.

Every minimal Lagrangian surface in a pseudo-Kähler 4-manifold is unstable [2]. The following Corollary explores the Hamiltonian stability of $G^{-}$-minimal Lagrangian surfaces in $\Sigma_{1} \times \Sigma_{2}$ :

Corollary 4. Let $\left(\Sigma_{1}, g_{1}\right)$ and $\left(\Sigma_{2}, g_{2}\right)$ be Riemannian two manifolds such that their Gauss curvatures $\kappa\left(g_{1}\right)$ and $\kappa\left(g_{2}\right)$ are both negative. Then every $G^{-}$-minimal Lagrangian surface is a local minimizer of the volume in its Hamiltonian isotopy class.

Proof. From Theorem 3 every $G^{-}$-minimal Lagrangian immersion must be of projected rank one and thus it is parametrised by $\Phi=(\phi, \psi): S \rightarrow \Sigma_{1} \times \Sigma_{2}$, where $\phi=\phi(s)$ and $\psi=\psi(t)$, where $s, t$ are arclengths. Assuming that $\kappa\left(g_{1}\right), \kappa\left(g_{2}\right)$ are both negative we have that:

$$
\kappa\left(g_{1}\right)(s) \leq-2 k_{\phi}^{2}(s)=0, \quad \kappa\left(g_{2}\right)(t) \leq-2 k_{\psi}^{2}(t)=0,
$$

and therefore from Theorem 4 the $G^{-}$-minimal Lagrangian immersion $\Phi$ is stable under Hamiltonian deformations.

We also have the Corollary:

Corollary 5. Let $(\Sigma, g)$ be a Riemannian two manifold of negative Gaussian curvature. Then every $G^{-}$-minimal Lagrangian surface immersed in $\Sigma \times \Sigma$ is a local minimizer of the volume in its Hamiltonian isotopy class.

Example 1. It is easy to see that if $(\Sigma, g)$ is a Riemannian two manifold of constant Gauss curvature $c \neq 0$, then every $G^{-}$-minimal Lagrangian surface immersed in $\Sigma \times \Sigma$ is a local minimizer of the volume in its Hamiltonian isotopy class if and only if $c<0$. 
Example 2. Let $L\left(\mathbb{S}^{3}\right)$ and $L^{+}\left(A d \mathbb{S}^{3}\right)$ be the spaces of oriented closed geodesics in the three sphere and anti-De Sitter 3-space, respectively. Then $L\left(\mathbb{S}^{3}\right)=\mathbb{S}^{2} \times \mathbb{S}^{2}$ and $L^{+}\left(A d \mathbb{S}^{3}\right)=\mathbb{H}^{2} \times \mathbb{H}^{2}$ (see [1] and [3]). The previous example generalises a result obtained in [5] which states that every minimal Lagrangian surface in the space of closed oriented geodesics $L\left(\mathbb{S}^{3}\right)$ is Hamiltonian unstable and every Lagrangian minimal surface in $L^{+}\left(A d \mathbb{S}^{3}\right)$ is Hamiltonian stable.

The following Proposition investigates the Hamiltonian stability of $G^{+}$-minimal Lagrangian surfaces:

Proposition 3. Let $\left(\Sigma_{1}, g_{1}\right)$ and $\left(\Sigma_{2}, g_{2}\right)$ be Riemannian two manifolds with Gaussian curvatures bounded in the following way:

$$
c_{1} \leq\left|\kappa\left(g_{1}\right)(x)\right| \leq C_{1}, \quad c_{2} \leq\left|\kappa\left(g_{2}\right)(y)\right| \leq C_{2}, \quad \text { and } \quad \kappa\left(g_{1}\right)(x) \kappa\left(g_{2}\right)(y)<0,
$$

for every pair $(x, y) \in \Sigma_{1} \times \Sigma_{2}$ and for some positive constants $c_{1}, c_{2}, C_{1}, C_{2}$. Then, every $G^{+}$-minimal Lagrangian surface is Hamiltonian unstable and therefore is $G^{+}$. unstable.

Proof. Consider again a Lagrangian minimal immersion $\Phi=(\phi, \psi): S \rightarrow \Sigma_{1} \times \Sigma_{2}$. From Theorem 3, we have that $\phi=\phi(s)$ and $\psi=\psi(t)$ are geodesics of $\Sigma_{1}$ and $\Sigma_{2}$, respectively, with $(s, t)$ chosen to be the corresponding arc-lengths.

Then $\left(\phi_{s}, j_{1} \phi_{s}\right)$ is an oriented orthonormal frame of $\left(\Sigma_{1}, g_{1}\right)$ and $\left(\psi_{t}, j_{2} \psi_{t}\right)$ is an oriented orthonormal frame of $\left(\Sigma_{2}, g_{2}\right)$.

A similar computation as in Theorem 4 gives,

$$
\operatorname{Ric}^{+}\left(\Phi_{s}, \Phi_{s}\right)=\kappa\left(g_{1}\right), \quad \operatorname{Ric}^{+}\left(\Phi_{t}, \Phi_{t}\right)=\kappa\left(g_{2}\right), \quad \operatorname{Ric}^{+}\left(\Phi_{s}, \Phi_{t}\right)=0,
$$

and therefore, the second variation formula for the volume of $S$ in the direction of the Hamiltonian vector field $X=J \nabla u$ is

$$
\delta^{2} V(S)(X)=\int_{S}\left(\left(u_{s s}-u_{t t}\right)^{2}-\kappa\left(g_{1}\right) u_{s}^{2}-\kappa\left(g_{2}\right) u_{t}^{2}\right) d V .
$$

Assume that $\kappa\left(g_{1}\right)<0$. Then, $\kappa\left(g_{2}\right)>0$ and

$$
\delta^{2} V(S)(X) \geq \int_{S}\left(\left(u_{s s}-u_{t t}\right)^{2}-C_{1} u_{s}^{2}+c_{2} u_{t}^{2}\right) d V .
$$

Thus, for the quadratic functional

$$
Q_{1}(u):=\int_{S}-C_{1} u_{s}^{2}+c_{2} u_{t}^{2}
$$


there exists $u^{1} \in C_{c}^{\infty}(S)$ such that $Q_{1}\left(u^{1}\right) \geq 0$. Therefore $\delta^{2} V(S)\left(J \nabla u^{1}\right) \geq 0$. On the other hand, for every $u \in C_{c}^{\infty}(S)$

$$
\delta^{2} V(S)(J \nabla u) \leq \int_{S}\left(\left(u_{s s}+u_{t t}\right)^{2}-c_{1} u_{s}^{2}+C_{2} u_{t}^{2}\right) d V .
$$

Then for the quadratic functional

$$
Q_{2}(u):=\int_{S}-c_{1} u_{s}^{2}+C_{2} u_{t}^{2}
$$

there exists $u^{2} \in C_{c}^{\infty}(S)$ such that $Q_{2}\left(u^{2}\right) \leq 0$. A similar argument as in the proof of Theorem 3 of [5] establishes the existence of $u^{3} \in C_{c}^{\infty}(S)$ such that

$$
\int_{S}\left(\left(u_{s s}^{3}+u_{t t}^{3}\right)^{2}-c_{1}\left(u_{s}^{3}\right)^{2}+C_{2}\left(u_{t}^{3}\right)^{2}\right) d V \leq 0
$$

which implies that $\delta^{2} V(S)\left(J \nabla u^{3}\right) \leq 0$ and therefore the second variation formula for the volume of $S$ under Hamiltonian deformations is indefinite.

\section{Appendix}

The equation (5) has been proved in [10] (see also [8] for an alternative proof of it) for the case of a Lagrangian submanifold immersed in a Riemannian Kähler manifold. In the Appendix, we show that the same equation holds true for Lagrangian immersions in a neutral Käher 4-manifold.

Suppose now that $\Phi: S \rightarrow M^{4}$ is a smooth Lagrangian immersion of a surface $S$ in a neutral Kähler 4-manifold $(M, J, G, \Omega)$. Let $\left(e_{1}, e_{2}\right)$ be an orthonormal frame of the induced metric $\Phi^{*} G$ such that $\left|e_{1}\right|^{2}=-\left|e_{2}\right|^{2}=1$. The Lagrangian condition implies that $\left(e_{1}, e_{2}, J e_{1}, J e_{2}\right)$ is an orthonormal frame of $G$. If $h$ is the second fundamental form of $\Phi$ and $H=\frac{1}{2}\left(h\left(e_{1}, e_{1}\right)-h\left(e_{2}, e_{2}\right)\right)$ is the mean curvature vector, we consider the Maslov 1-form $a_{H}$ of $S$, defined by

$$
a_{H}:=G(J H, .)
$$

Proposition 4. Let $\Phi$ be a Lagrangian immersion in a neutral Kähler 4-manifold $(M, J, G, \Omega)$ and let $a_{H}$ be its Maslov 1-form. Then,

$$
d a_{H}=\Phi^{*} \bar{\rho}
$$

where $\bar{\rho}$ is the Ricci form of $G$. 
Proof. A straightforward computation gives:

$$
2 \Phi^{*} \bar{\rho}\left(e_{1}, e_{2}\right)=\overline{\operatorname{Ric}}\left(e_{1}, J e_{2}\right)=-G\left(\bar{R}\left(e_{1}, e_{2}\right) e_{1}, J e_{1}\right)+G\left(\bar{R}\left(e_{1}, e_{2}\right) e_{2}, J e_{2}\right)
$$

The Codazzi-Mainardi equations are:

$$
\begin{aligned}
& \perp \bar{R}\left(e_{1}, e_{2}\right) e_{1}=\bar{\nabla}_{e_{1}} h\left(e_{1}, e_{2}\right)-h\left(\nabla_{e_{1}} e_{1}, e_{2}\right)-h\left(\nabla_{e_{1}} e_{2}, e_{1}\right)-\bar{\nabla}_{e_{2}} h\left(e_{1}, e_{1}\right)+2 h\left(\nabla_{e_{2}} e_{1}, e_{1}\right), \\
& \perp \bar{R}\left(e_{1}, e_{2}\right) e_{2}=\bar{\nabla}_{e_{1}} h\left(e_{2}, e_{2}\right)-2 h\left(\nabla_{e_{1}} e_{2}, e_{2}\right)+h\left(\nabla_{e_{2}} e_{1}, e_{2}\right)-\bar{\nabla}_{e_{2}} h\left(e_{1}, e_{2}\right)+h\left(\nabla_{e_{2}} e_{2}, e_{1}\right),
\end{aligned}
$$

where $\bar{\nabla}$ and $\nabla$ denote the Levi-Civita connection with respect to $G$ and $\Phi^{*} G$, respectively.

Then,

$$
\begin{aligned}
2 \Phi^{*} \bar{\rho}\left(e_{1}, e_{2}\right)= & -G\left(\bar{R}\left(e_{1}, e_{2}\right) e_{1}, J e_{1}\right)+G\left(\bar{R}\left(e_{1}, e_{2}\right) e_{2}, J e_{2}\right) \\
= & -G\left(\bar{\nabla}_{e_{1}} h\left(e_{1}, e_{2}\right), J e_{1}\right)+G\left(h\left(\nabla_{e_{1}} e_{1}, e_{2}\right), J e_{1}\right)+G\left(h\left(\nabla_{e_{1}} e_{2}, e_{1}\right), J e_{1}\right) \\
& +G\left(\bar{\nabla}_{e_{2}} h\left(e_{1}, e_{1}\right), J e_{1}\right)-2 G\left(h\left(\nabla_{e_{2}} e_{1}, e_{1}\right), J e_{1}\right) \\
& +G\left(\bar{\nabla}_{e_{1}} h\left(e_{2}, e_{2}\right), J e_{2}\right)-2 G\left(h\left(\nabla_{e_{1}} e_{2}, e_{2}\right), J e_{2}\right)+G\left(h\left(\nabla_{e_{2}} e_{1}, e_{2}\right), J e_{2}\right) \\
& -G\left(\bar{\nabla}_{e_{2}} h\left(e_{1}, e_{2}\right), J e_{2}\right)+G\left(h\left(\nabla_{e_{2}} e_{2}, e_{1}\right), J e_{2}\right) \\
= & G\left(\bar{\nabla}_{e_{2}} h\left(e_{1}, e_{1}\right), J e_{1}\right)-G\left(\bar{\nabla}_{e_{1}} h\left(e_{1}, e_{2}\right), J e_{1}\right)+G\left(\bar{\nabla}_{e_{1}} h\left(e_{2}, e_{2}\right), J e_{2}\right) \\
& -G\left(\bar{\nabla}_{e_{2}} h\left(e_{1}, e_{2}\right), J e_{2}\right)+G\left(h\left(e_{1}, e_{2}\right), J \nabla_{e_{1}} e_{1}\right)+G\left(h\left(e_{1}, e_{1}\right), J \nabla_{e_{1}} e_{2}\right) \\
& \quad-2 G\left(h\left(e_{1}, e_{1}\right), J \nabla_{e_{2}} e_{1}\right)-2 G\left(h\left(e_{2}, e_{2}\right), J \nabla_{e_{1}} e_{2}\right) \\
& +G\left(h\left(e_{2}, e_{2}\right), J \nabla_{e_{2}} e_{1}\right)+G\left(h\left(e_{1}, e_{2}\right), J \nabla_{e_{2}} e_{2}\right) .
\end{aligned}
$$

Set

$$
A:=G\left(\bar{\nabla}_{e_{2}} h\left(e_{1}, e_{1}\right), J e_{1}\right)-G\left(\bar{\nabla}_{e_{1}} h\left(e_{1}, e_{2}\right), J e_{1}\right)+G\left(\bar{\nabla}_{e_{1}} h\left(e_{2}, e_{2}\right), J e_{2}\right)-G\left(\bar{\nabla}_{e_{2}} h\left(e_{1}, e_{2}\right), J e_{2}\right)
$$

so that,

$$
\begin{aligned}
& 2 \Phi^{*} \bar{\rho}\left(e_{1}, e_{2}\right)= A+G\left(h\left(e_{1}, e_{2}\right), J \nabla_{e_{1}} e_{1}\right)+G\left(h\left(e_{1}, e_{1}\right), J\left[e_{1}, e_{2}\right]\right) \\
&-G\left(h\left(e_{1}, e_{1}\right), J \nabla_{e_{2}} e_{1}\right)-G\left(h\left(e_{2}, e_{2}\right), J \nabla_{e_{1}} e_{2}\right) \\
&+G\left(h\left(e_{2}, e_{2}\right), J\left[e_{2}, e_{1}\right]\right)+G\left(h\left(e_{1}, e_{2}\right), J \nabla_{e_{2}} e_{2}\right) \\
&=A+G\left(2 H, J\left[e_{1}, e_{2}\right]\right)+G\left(h\left(e_{1}, e_{2}\right), J \nabla_{e_{1}} e_{1}\right) \\
& \quad-G\left(h\left(e_{1}, e_{1}\right), J \nabla_{e_{2}} e_{1}\right)-G\left(h\left(e_{2}, e_{2}\right), J \nabla_{e_{1}} e_{2}\right)+G\left(h\left(e_{1}, e_{2}\right), J \nabla_{e_{2}} e_{2}\right) \\
&=2 A-2 G\left(J H,\left[e_{1}, e_{2}\right]\right)+\bar{\nabla}_{e_{1}} G\left(h\left(e_{1}, e_{1}\right), J e_{2}\right)-\bar{\nabla}_{e_{2}} G\left(h\left(e_{1}, e_{1}\right), J e_{1}\right) \\
&+\bar{\nabla}_{e_{2}} G\left(h\left(e_{2}, e_{2}\right), J e_{1}\right)-\bar{\nabla}_{e_{1}} G\left(h\left(e_{2}, e_{2}\right), J e_{2}\right) .
\end{aligned}
$$


Note that,

$$
\begin{aligned}
A=\bar{\nabla}_{e_{2}} G & \left(h\left(e_{1}, e_{1}\right), J e_{1}\right)-G\left(h\left(e_{1}, e_{1}\right), J \nabla_{e_{2}} e_{1}\right) \\
- & \bar{\nabla}_{e_{1}} G\left(h\left(e_{1}, e_{1}\right), J e_{2}\right)+G\left(h\left(e_{1}, e_{2}\right), J \nabla_{e_{1}} e_{1}\right) \\
+ & \bar{\nabla}_{e_{1}} G\left(h\left(e_{2}, e_{2}\right), J e_{2}\right)-G\left(h\left(e_{2}, e_{2}\right), J \nabla_{e_{1}} e_{2}\right) \\
& \quad-\bar{\nabla}_{e_{2}} G\left(h\left(e_{2}, e_{2}\right), J e_{1}\right)+G\left(h\left(e_{1}, e_{2}\right), J \nabla_{e_{2}} e_{2}\right) .
\end{aligned}
$$

We now have,

$$
\begin{aligned}
2 \Phi^{*} \bar{\rho}\left(e_{1}, e_{2}\right)= & 2 A-2 G\left(J H,\left[e_{1}, e_{2}\right]\right)+\bar{\nabla}_{e_{1}} G\left(h\left(e_{1}, e_{1}\right), J e_{2}\right)-\bar{\nabla}_{e_{2}} G\left(h\left(e_{1}, e_{1}\right), J e_{1}\right) \\
& \quad+\bar{\nabla}_{e_{2}} G\left(h\left(e_{2}, e_{2}\right), J e_{1}\right)-\bar{\nabla}_{e_{1}} G\left(h\left(e_{2}, e_{2}\right), J e_{2}\right) \\
= & -2 G\left(J H,\left[e_{1}, e_{2}\right]\right)+\bar{\nabla}_{e_{2}} G\left(h\left(e_{1}, e_{1}\right), J e_{1}\right)-\bar{\nabla}_{e_{1}} G\left(h\left(e_{1}, e_{1}\right), J e_{2}\right) \\
& +\bar{\nabla}_{e_{1}} G\left(h\left(e_{2}, e_{2}\right), J e_{2}\right)-\bar{\nabla}_{e_{2}} G\left(h\left(e_{2}, e_{2}\right), J e_{1}\right)-2 G\left(h\left(e_{1}, e_{1}\right), J \nabla_{e_{2}} e_{1}\right) \\
& +2 G\left(h\left(e_{1}, e_{2}\right), J \nabla_{e_{1}} e_{1}\right)-2 G\left(h\left(e_{2}, e_{2}\right), J \nabla_{e_{1}} e_{2}\right)+2 G\left(h\left(e_{1}, e_{2}\right), J \nabla_{e_{2}} e_{2}\right) \\
= & \bar{\nabla}_{e_{2}} G\left(2 H, J e_{1}\right)-\bar{\nabla}_{e_{1}} G\left(2 H, J e_{2}\right)-2 G\left(J H,\left[e_{1}, e_{2}\right]\right)-2 G\left(h\left(e_{1}, e_{1}\right), J \nabla_{e_{2}} e_{1}\right) \\
& +2 G\left(h\left(e_{1}, e_{2}\right), J \nabla_{e_{1}} e_{1}\right)-2 G\left(h\left(e_{2}, e_{2}\right), J \nabla_{e_{1}} e_{2}\right)+2 G\left(h\left(e_{1}, e_{2}\right), J \nabla_{e_{2}} e_{2}\right) \\
= & 2 \bar{\nabla}_{e_{1}} G\left(J H, e_{2}\right)-2 \bar{\nabla}_{e_{2}} G\left(J H, e_{1}\right)-2 G\left(J H,\left[e_{1}, e_{2}\right]\right)-2 G\left(h\left(e_{1}, e_{1}\right), J \nabla_{e_{2}} e_{1}\right) \\
& +2 G\left(h\left(e_{1}, e_{2}\right), J \nabla_{e_{1}} e_{1}\right)-2 G\left(h\left(e_{2}, e_{2}\right), J \nabla_{e_{1}} e_{2}\right)+2 G\left(h\left(e_{1}, e_{2}\right), J \nabla_{e_{2}} e_{2}\right) \\
= & 2 d a_{H}\left(e_{1}, e_{2}\right)-2 G\left(h\left(e_{1}, e_{1}\right), J \nabla_{e_{2}} e_{1}\right)+2 G\left(h\left(e_{1}, e_{2}\right), J \nabla_{e_{1}} e_{1}\right)
\end{aligned}
$$

Let $\omega_{i j}^{k}$ be the functions defined by

$$
\nabla_{e_{i}} e_{j}=\sum_{k=1}^{2} \omega_{i j}^{k} e_{k}
$$

Since $\left|e_{1}\right|^{2}=-\left|e_{2}\right|^{2}=1$, we have

$$
\omega_{11}^{2}=\omega_{12}^{1}, \quad \omega_{21}^{2}=\omega_{22}^{1}
$$

from which we deduce that

$$
G\left(h\left(e_{1}, e_{1}\right), J \nabla_{e_{2}} e_{1}\right)=G\left(h\left(e_{1}, e_{2}\right), J \nabla_{e_{2}} e_{2}\right),
$$

and

$$
G\left(h\left(e_{1}, e_{2}\right), J \nabla_{e_{1}} e_{1}\right)=G\left(h\left(e_{2}, e_{2}\right), J \nabla_{e_{1}} e_{2}\right) .
$$

The proposition follows by substituting (11) and (12) into (10). 


\section{References}

[1] D. Alekseevsky, B. Guilfoyle, W. Klingenberg, On the geometry of spaces of oriented geodesics, Ann. Global Anal. Geom. 40 (2011) 1-21

[2] H. Anciaux, Minimal submanifolds in pseudo-Riemannian geometry, World Scientific, (2010)

[3] H. Anciaux, Space of geodesics of pseudo-Riemannian space forms and normal congruences of hypersurfaces, to appear in Transactions of the AMS

[4] H. Anciaux, I. Castro, Construction of Hamiltonian-minimal Lagrangian submanifolds in complex Euclidean space, Results in Math. 60 (2011) $325-349$

[5] H. Anciaux, N. Georgiou, Hamiltonian stability of Hamiltonian minimal Lagrangian submanifolds in pseudo- and para- Kähler manifolds, Adv. in Geom. (to appear)

[6] H. Anciaux, B. Guilfoyle, P. Romon, Minimal submanifolds in the tangent bundle of a Riemannian surface, J. Geometry and Physics. 61 (2011) $237-247$

[7] H. Anciaux, P. Romon, A canonical structure of the tangent bundle of a pseudo- or para- Kähler manifold, arxiv:1301.4638

[8] R.L. Bryant, Minimal Lagrangian submanifolds of Kähler-Einstein manifolds, Chaohao, G. et al. (eds.) Differential Geometry and Differential Equations. Lect. Notes Math., 1255 Berlin Heidelberg New York: Springer (1987), 1-12

[9] I. Castro, F. Torralbo, F. Urbano, On Hamiltonian stationary Lagrangian spheres in non-Einstein Kähler surfaces, Math. Z. 271 (2012) 259-270

[10] P. Dazord, Sur la gómetrie des sous-fibrés et des feuilletages lagrangiense, Ann. Sci. Éc. Norm. Super., IV. Ser. 13, (1981) 465-480

[11] A. Derdziński, Self-dual Kähler manifolds and Einstein manifolds of dimension four, Compositio Math 49(3), (1983) 405-433 
[12] N. Georgiou, On area stationary surfaces in the space of oriented geodesics of hyperbolic 3-space, Math. Scand. 111 (2012) 187-209

[13] N. Georgiou, B. Guilfoyle, On the space of oriented geodesics of hyperbolic 3-space, Rocky Mountain J. Math. 40 (2010) 1183-1219

[14] B. Guilfoyle, W. Klingenberg, An indefinite $K$ ahler metric on the space of oriented lines, J. London Math. Soc. 72 (2005), 497-509

[15] R. Harvey and H.B. Lawson, Calibrated geometries, Acta Math. 148(1982) 47-157

[16] Y.G. Oh, Second variation and stabilities of minimal lagrangian submanifolds in Kähler manifolds, Invent. Math. 101(1990) 501-519

[17] Y.G. Oh, Volume minimization of Lagrangian submanifolds under Hamiltonian deformations, Math. Z. 212(1993) 175-192

[18] M. Salvai, On the geometry of the space of oriented lines in Euclidean space, Manuscripta Math. 118 (2005), 181-189

[19] M. Salvai, On the geometry of the space of oriented lines of hyperbolic space, Glasg. Math. J. 49 (2007), 357-366

[20] J. Simons, Minimal varieties in Riemannian manifolds Ann. Math. 88(1968) 82-105

[21] A. Strominger, S.-T. Yau, E. Zaslow, Mirror symmetry is T-duality, Nuclear Phys. B479(1996) 243-259

[22] F. Urbano, Hamiltonian stability and index of minimal Lagrangian surfaces in complex projective plane, Indiana Univ. Math. J. 56(2007) 931946

Department of Mathematics and Statistics, University of Cyprus, P.O. Box 20537, 1678 Nicosia, Cyprus

E-mail: georgiou.g.nicos@ucy.ac.cy 